https://jurnal.unimed.ac.id/2012/index.php/studia/index

\title{
DIE FEHLERANALYSE BEI DER ANWENDUNG DER KONJUNKTIONEN (ALS UND WENN) BEI DEN STUDENTEN \\ IM DRITTEN SEMESTER
}

\author{
Meilyn Sarah Pangaribuan \\ Ahmad Sahat Perdamean \\ Linda Aruan
}

\begin{abstract}
ABSTRAKT
Das Ziel dieser Untersuchung ist die Fehler von den Deutschstudenten im dritten Semester im Jahrgang 2017/2018 bei der Anwendung der Konjunktionen ,als” und ,wenn” zu analysieren. In dieser Untersuchung wird deskriptive quantitative Methode angewendet. Die Population in dieser Untersuchung sind die Deutschstudenten im dritten Semester im Jahrgang 2017/2018, der aus 3 Klassen besteht. Die Probanden sind alle Studenten im dritten Semester von drei Klassen, Regulärklasse und Extensionklasse, die aus 21 Stundenten A Regulärklasse, 23 Studenten B Regulärklasse und 12 Studenten Extensionklasse besteht. Die Deutschstudenten sollen die Sätze mit den Konjunktionen ,als” oder „wenn” ergänzen. Basierend auf der Analyse werden zwei Arten der Fehler der Deutschstudenten gefunden, sie sind: (1) die Konjunktion ,,als” (189 Fehler, 48,71\%), (2) die Konjunktion ,wenn” (199 Fehler, 51,28\%). Ausgehend von der Fehleranalyse sind die meisten Fehler bezüglich der Konjunktion ,wenn” (199 Fehler, 51,28\%).

Schlüsselwörter : Fehleranalyse, Konjunktion ,als” und Konjunktion „,wenn”.
\end{abstract}

\section{EINLEITUNG}

Sprache ist sehr wichtig für das menschliche Leben. Ohne Sprache kann man mit anderen Leuten nicht richtig kommunizieren. Chaer (2005:33) behauptet, dass Sprache eine soziale Interaktion ist, die eine Symbol und eine Klangform haben. Aus diesem Grund ist die Sprache ein wichtiges System sprachlicher Zeichen und Verständigung für das menschliche Leben, die gelernt werden muss. Das ist genauso mit Fremdsprachen.

Heutzutage sind Fremdsprachen am populärsten in der Erziehung. Die Fremdsprache wird nicht nur von Schülern, sondern auch von Studenten gelernt. Eine von vielen wichtigen Fremdsprachen ist deutsch. Deutsch wird in vielen Universitäten in Indonesien gelernt. Wenn man eine Fremdsprache lernt, muss man die Kompetenz der Fremdsprache lernen. Deutsch hat vier Kompetenzen, die beherrscht und entwickelt werden müssen. Diese Kompetenzen sind Hörverstehen, Sprechfertigkeit, Schreibfertigkeit und Leseverstehen. Außer diesen vier Kompetenzen lernt man auch 
Grammatik, weil die Grammatik das Grundgerüst einer Sprache ist und bestimmte Regeln vorgibt. Das Lernen der Grammatik ist eine der Schwierigkeiten Aufgaben beim Lernen einer Fremdsprache. Man muss Grammatikkenntnisse beherrschen, damit man einen richtigen Satz formen kann und das hat nichts mit der Grammatik zu tun. Konjunktionen sind ein teil der Grammatik. Die Konjunktionen ,als” und „wenn” sollen in dieser Untersuchung genauer betrachtet werden.

Die Konjunktionen ,als” und „,wenn” werden von der Studenten im dritten Semester gelernt. Basierend auf Interviews mit den Studenten wird herausgefunden, dass die Konjunktionen ,als” und „wenn” die gleiche Bedeutung haben, deshalb machen viele Studenten Fehler bei ihrer Verwendung. Die Studenten behaupten, dass sie die Anwendung der Konjuntionen ,als” und „wenn” nicht verstehen. Die Studenten können die richtige Position des Verbs bei der Verwendung der Konjunktionen ,als” und „wenn” im Satz nicht bestimmen. Daher ist es wichtig, diesen Teil der Grammatik sorgfältig zu lernen, damit die Fehler minimiert werden können.

Die Fehler, die die Studenten im dritten Semester gemacht haben, werden untersucht. Wenn die Konjunktionen ,,als” und ,wenn” in einem Satz benutzt wurden, hatten die Studenten Schwierigkeiten.

Ein Beispiel für einen Fehler bei der Anwendung der Konjunktion ,als” ist folgendes:

\section{Als ich viel Geld habe, kaufe ich ein Buch.}

Die Konjunktion ,als” ist in diesem Satz falsch benutzt worden.

Der richtige Satz lautet: Wenn ich viel Geld habe, kaufe ich ein Buch.

Ein Beispiel für einen Fehler bei der Anwendung der Konjunktion „wenn” ist folgendes:

Wenn ich 11 Jahre alt war, flog ich nach Deutschland.

Die Konjunktion „,wenn” ist in diesem Satz falsch benutzt worden.

Der richtige Satz lautet: Als ich 11 Jahre alt war, flog ich nach Deutschland.

Aus den oben genannten Fehlern, die die Studenten im Satz gemacht haben, kann geschlussfolger werden, dass die Studenten die Anwendung der Konjunktionen ,,als” und ,wenn” im Satz noch nicht richtig verstanden haben und daher Probleme bei der Bildung von Sätzen mit diesen Konjunktionen haben. 


\section{THEORETISCHE GRUNDLAGE}

\section{Der Begriff der Fehleranalyse}

Beim Fremdsprachenlernen macht man oft Fehler. Fehler können beim Erlernen einer Sprache nicht vermieden werden. Diese Fehler können zum Problem werden, wenn die Person den Fehler nicht versteht oder sich des Fehlers beim Sprechen nicht bewusst ist. Veijonen (2008:1) behauptet, dass die Fehleranalyse ein Teil des Fremdsprachenunterrichts und des Fremdsprachenlernens geworden ist. Man macht aber keine Fehleranalyse nur um herauszufinden, wie viele oder welche Fehler die Lerner machen, sondern man will auch einen Nutzen von der Analyse haben. Die Fehleranalyse ist nützlich in vieler Hinsicht, zum Beispiel können Lehrbuchautoren, die daran denken müssen, welche Sachverhalte sie in den Büchern mehr als andere betonen wollen, von fehleranalytischen Untersuchungen profitieren. Auch sowohl der Lerner als auch der Lehrer können einen Nutzen aus der Fehleranalyse ziehen.

Häufig kommt es beim Schreiben zu Fehlern. Der Grund dafür ist mangelnde Genauigkeit beim Schreiben. Ellis in Tarigan (2011:153) schreibt, dass die Fehleranalyse ein Arbeitsverfahren von Sprachverfassern und Sprachlehrern ist. Die Fehleranalyse kann mehrere Vorteile bringen. Das Verständis der Fehler kann zur Evaluation benutzt werden, die im nachhinein Verbesserungen beim Lernen bringen kann (vgl. Tarigan, 2011:61).

Basierend auf den zuvor genannten Expertenmeinungen kann zusammengefasst werden, dass die Fehleranalyse ein Arbeitsverfahren und ein Arbeitsprozess von Sprachverfassern und Sprachlehrern ist, die als Teil des Fremdsprachenunterrichts und des Fremdsprachenlernens benutzt wird und Verbesserungen beim Lernen bringen kann.

\section{Die Ziele der Fehleranalyse}

Corder in Veijonen (2008:5) findet, dass eine Fehleranalyse folgende Ziele hat:

a. Der Lehrer bekommt Information darüber, wie weit der Lerner gekommen ist und was er noch zu lernen hat.

b. Die Verfasserin interessiert sich für Fehler, weil es Beweise dafür gibt, wie die Sprache gelernt wird und welche Strategien der Lerner beim Lernen benutzt. 
c. Der Lerner wird darüber informiert, wie gut er die Sprache gelernt hat. Dem Lerner wird klar gemacht, dass Fehler beim Lernen notwendig sind und für den Lernprozess hilfreich sein können.

Sidhar in Tarigan (2011:61) stellt fest, dass die Fehleranalyse darüber hinaus folgende Ziele hat:

a. Sie kann das Lehrmaterial im Klassenzimmer und in den Lehrbüchern bestimmen, zum Beispiel den Übergang von einem leichten Thema zu einem schwierigen.

b. Sie kann die Reihenfolge der Erklärungen und Übungen im Unterricht bestimmen.

c. Der Lehrer kann durch sie Übungen und Wiederholungen planen.

d. Durch sie können die passenden Aufgaben für Tests ausgewählt werden.

\section{Die Phasen der Fehleranalyse}

Fehler, die in der Sprache gemacht werden, können das Erreichen der Ziele des Sprachunterrichts aber auch stören. Deshalb sollten die Fehler verringert oder beseitigt werden. Corder in Veijonen (2008:19) erklärt, dass die Phasen der Fehleranalyse folgendermaßen eingeteilt werden:

a. Die Identifizierung

b. Die Klassifizierung

c. Die Erklärung

Tarigan und Tarigan (2011:60) behaupten, dass die Fehleranalyse auch folgendermaßen ablaufen kann:
a. Proben sammeln
b. Die Fehler identifizieren
c. Die Fehler beschreiben
d. Die Fehler klassifizieren
e. Die Fehler evaluieren

Man kann also zusammenfassen, dass Fehler zuerst identifiziert, beschreiben und anschließend klassifiziert und evaluiert werden müssen.

\section{Der Begriff der Konjunktionen}

Konjunktionen sind Teile der Grammatik, die von den Deutschstudenten gelernt werden. Götze (2004:314) argumentiert, dass Konjunktionen unveränderliche 
Wörter sind. Die Konjunktionen sind weder Satzglieder noch Attribute, sondern verbinden Wörter, Teile von Sätzen oder ganze Sätze miteinander. Konjunktionen sind also ein wichtiges Mittel der Verbindung und der Koordination von Satzelementen.

Götze (2004:315) behauptet, dass es etwa 70 bis 80 Konjunktionen im Deutschen gibt, wie zum Beispiel „als”, „und”, „als dass”, „aber”, „wenn”, „aber”, „denn”, „bevor”, „dam” , usw. Götze (2004:315) erklärt, dass es 17 verschiedene Typen von Konjunktionen gibt.

1. Koordinierende Konjunktion (zum Beispiel „aber”, „doch”, ,jedoch”, „,sondern”, „,so(wie)”, ,entweder....oder”, „,nicht nur....sondern auch”, „,so....wie”, „,sowohl....als auch”, ,weder....noch”, ,, zwar....aber”)

2. Kopulative Konjunktion (zum Beispiel „, und”)

3. Die Kausale (begründende) Konjunktion (zum Beispiel „denn”, „,weil”)

4. Alternative (ausschließende) Konjunktion (zum Beispiel „oder”, „weder....noch”, ,,entweder....oder”, „,bzw”, ,aber”)

5. Adversative Konjunktion (zum Beispiel „allein”, „sondern”, „zwar....aber")

6. Konkretisierende Konjunktion (zum Beispiel „d. h”)

7. Satzteil Konjunktion (zum Beispiel „,wie”, „umso”)

8. Subordinierende Konjunktion (zum Beispiel „als”, „bevor”, „bis”, „da”, „damit”, „dass”, „,ehe”, ,falls”, ,indem”, „,nachdem”, „,ob”, „obgleich”, „,obwohl”, „seit(dem)”, „sobald”, „solange”, „während”, „weil”, „wenn”, „wenn auch”, „zumal”, ,als dass”, „als ob”, ,als wenn”, „außer dass” , ,außer wenn”, „kaum dass”, „ohne dass”, „so dass”, ,,wenn auch....so doch”)

9. Konsekutive (folgernde) Konjunktion (zum Beispiel „so dass”, „ohne”)

10. Konditionale (bedingende) Konjunktion (zum Beispiel „wenn”)

11. Konzessive (Einräumende) Konjunktion (zum Beispiel „,obwohl”, „,trotzdem”)

12. Modale Konjunktion (zum Beispiel „,in dem”, „dadurch....dass”)

13. Temporale Konjunktion (zum Beispiel ,,indem”, „,nachdem”, „,bevor/ehe”)

14. Vergleichende Konjunktion (zum Beispiel ,als ob”)

15. Restriktive (einschränkende) Konjunktion (zum Beispiel „soviel”, „,in sofern als")

16. Die Adversative Konjunktion (zum Beispiel ,,aber/jedoch”, „während”) 
17. Finale Konjunktion (zum Beispiel „damit”, „außer”)

\section{Die Anwendung der Konjunktionen ,als” und „wenn”}

Auf der Seite http://www.mein-deutschbuch.de/ wird die die Anwendung der Konjunktionen ,als” und ,wenn” beschrieben und hier zitiert:

„Temporalsätze mit den Konjunktionen ,als” und „wenn” drücken eine Gleichzeitigkeit zweier Handlungen zu einem Zeitpunkt aus. Das passende Fragewort lautet „Wann”?” „Die Konjunktion „wenn” benutzt man für eine gleichzeitige Handlung in der Zukunft und in der Gegenwart sowie für eine wiederholte Handlung in der Vergangenheit. Die Konjunktion „als” wird nur für eine einmalige Handlung in der Vergangenheit benutzt."

Tabelle 1 Die Anwendung der Konjunktionen „als” und „wenn”

\begin{tabular}{|c|c|c|c|}
\hline Tempus & Fragewort & Konjunktion & Wie oft \\
\hline Zukunft & Wann? & $\begin{array}{c}\text { Wenn } \\
\text { (immer)wenn; } \\
\text { (jedes mal)wenn }\end{array}$ & $\begin{array}{c}\text { mehrmalige } \\
\text { Handlungen/ } \\
\text { einmalige } \\
\text { Handlungen }\end{array}$ \\
\hline Gegenwart & Wann? & $\begin{array}{c}\text { (immer)wenn; } \\
\text { (jedes mal)wenn }\end{array}$ & $\begin{array}{c}\text { mehrmalige } \\
\text { Handlungen }\end{array}$ \\
\hline Vergangenheit & Wann? & $\begin{array}{c}\text { (immer)wenn; } \\
\text { (jedes mal)wenn }\end{array}$ & $\begin{array}{c}\text { Handlungen } \\
\text { einmalige } \\
\text { Handlungen }\end{array}$ \\
\hline Vergangenheit & Wann? & als & \multicolumn{2}{|c|}{} \\
\hline
\end{tabular}

Tabelle 2 Die Anwendung der Konjunktionen „als" und „wenn” Nebensatz + Hauptsatz

\begin{tabular}{|l|l|}
\hline \multicolumn{1}{|c|}{ Nebensatz } & \multicolumn{1}{c|}{ Hauptsatz } \\
\hline Wenn die Dämmerung beginnt, & komme ich nach Hause. \\
\hline $\begin{array}{l}\text { (Immer) wenn Oma zu Besuch } \\
\text { kommt, }\end{array}$ & ist das Kind glücklich. \\
\hline $\begin{array}{l}\text { Wenn das Mädchen Liebeskummer } \\
\text { hatte, }\end{array}$ & hat es (jedes Mal) bitterlich geweint \\
\hline Als Rolf 28 Jahre alt war, & ist er ausgewandert. \\
\hline $\begin{array}{l}\text { Als Simon noch ein kleiner Junge } \\
\text { war, }\end{array}$ & sind seine Eltern gestorben. \\
\hline
\end{tabular}

\section{Die Untersuchungsmethode}

Diese Untersuchung benutzt die deskriptive quantitative Methode, so dass eine exakte Datenanalyse mit der Technik der Prozentanalyse erzielt wird. 
Um die Fehler der Studenten bei den Konjunktionen ,als” und „,wenn” zu beschreiben, wird das Untersuchungsergebnis an die Entfernungsformel von Gulo angepasst:

1. Wenn die Fehler $0 \%-25 \%$ betragen: gut (A)

2. Wenn die Fehler $26 \%-50 \%$ betragen: ausreichen (B)

3. Wenn die Fehler $51 \%-75 \%$ betragen: wenig (C)

4. Wenn die Fehler $76 \%-100 \%$ betragen: weniger (D)

Um die Fehler der Studenten im dritten Semester im Jahrgang 2017/2018 beim Konjunktionen ,als” und „wenn” anwenden, wird das Untersuchungsergebnis an den Bewertungsindex der Studenten an der Staatlichen Universität Medan (2007:121) anpasst:

$$
\begin{aligned}
& 90-100=\text { (A) sehr kompetent } \\
& 80-89=(\mathrm{B}) \text { kompetent } \\
& 70-79=(\mathrm{C}) \text { ausreichend } \\
& 0 \quad-69=(\mathrm{D}) \text { inkompetent }
\end{aligned}
$$

\section{Das Untersuchungsergebnis}

Die Fehler der Deutschstudenten im dritten Semester bei der Anwendung der Konjunktionen ,als" und ,wenn"

Dieses Kapitel erklärt die Ergebnisse der Analyse, das sind: a) die Fehler der Deutschstudenten im dritten Semester bei der Anwendung der Konjunktionen „,als” und „wenn”, b) die häufigsten Fehler der Deutschstudenten im dritten Semester bei der Anwendung der Konjunktionen ,als” und „wenn”, c) die Analyse der Fehler der Deutschstudenten im dritten Semester bei der Anwendung der Konjunktionen ,als” und ,wenn".

Die häufigsten Fehler der Deutschstudenten im dritten Semester bei der Anwendung der Konjunktionen ,als" und ,wenn”

Basierend auf der Analyse werden zwei Arten der Fehler gefunden, die von den Deutschstudenten bei der Anwendung der Konjunktionen ,als” und „wenn” gemacht werden. Die Fehler sind: 1) als, 2) wenn. Die Arten der Fehler der Deutschstudenten können in der Tabelle 4.1. gesehen werden.

Tabelle 3 Die Arten der Fehler

\begin{tabular}{|c|l|c|c|}
\hline Nr. & Die Arten der Fehler & Anzahl & Prozent (\%) \\
\hline 1. & Als & 189 & $48,71 \%$ \\
\hline
\end{tabular}


https://jurnal.unimed.ac.id/2012/index.php/studia/index

\begin{tabular}{|c|c|c|c|}
\hline 2. & Wenn & 199 & $51,28 \%$ \\
\hline & Summe & 388 & $100 \%$ \\
\hline
\end{tabular}

Die Fehleranalyse kann die Fehler der Deutschstudenten bei der Anwendung der Konjunktionen ,als" und ,wenn” darstellen. Es gibt 189 Fehler (48,71\%) betreffend der Konjunktion ,als”, 199 Fehler (51,28\%) betreffend der Konjunktion ,,wenn”.

Die Analyse der Fehler der Deutschstudenten im dritten Semester bei der Anwendung der Konjunktionen ,als” und ,wenn”

\section{Die Konjunktion ,,als"}

Fehler bei der Anwendung Konjunktion ,,als”, die die Deutschstudenten gemacht haben, gibt es insgesamt 189 (48,71\%). Viele Fehler haben die Deutschstudenten bei der Anwendung der Konjunktion „als” gemacht. Die Deutschstudenten verstehen die Anwendung der Zeitform der Konjunktionen ,als” nicht. Die Konjunktion ,,als” wird für Präteritum angewendet und die Konjunktion „als” wird für einmalige Handlungen benutzt. Die Deutschstudenten können zwischen der Bedeutung der Konjunktionen ,als” und „wenn” nicht unterscheiden. Die Deutschstudenten können zwischen den Adverbien für die Konjunktionen ,als” oder „,wenn” nicht unterscheiden. Die Adverbien der Konjunktion ,,als” sind „letztes Jahr”, „,das Jahr zuvor”, ,vor zwei Jahren”, „,erste Mal”, und zum Beispiel „,17 Jahre alt war”. Die Deutschstudenten verstehen die Bedeutung der Sätze nicht, die nur einmalige Handlungen oder mehrmalige Handlungen beschreiben. Deshalb machen die Deutschstudenten Fehler im Satz.

Diese Fehler gibt es in den Aufgaben Nummer 1,3,4,5,6 im ersten Teil, bei der Buchstaben a,c im zweiten Teil bei den Nummer 1,2,3,5,6 im dritten Teil. Die meisten Fehler werden bei Nummer 2 im dritten Teil (33 Fehler), die wenigsten Fehler in Aufgabe a,c im zweiten Teil und Nummer 3 im dritten Teil (6 Fehler) gemacht.

\section{Teil I}

1. Schon am ersten Tag, wir eine kleine Bergtour gemacht haben, ist mein Vater gestürzt und wir mussten ins Krankenhaus, in die Notaufnahme. (die Aufgabe Nummer 1). 
In dieser Aufgabe gab es 15 Personen, die falsch geantwortet haben. Die Deutschstunden haben mit der Konjunktion ,wenn” geantwortet und das ist falsch. In dieser Satz gibt es ein Adverb der Konjunktion ,als”. Das Adverb ist „,am ersten Tag” mit dem Konjunktion ,als” angewendet und mit Präteritum benutzt wird. Das ist eine einmalige Handlung. Obwohl in dieser Aufgabe das Partizip Perfekt benutzt wird muss die Konjunktion ,als” angewendet werden.

Die richtige Antwort ist:

Schon am ersten Tag, $\underline{\text { als }}$ wir eine kleine Bergtour gemacht haben, ist mein Vater gestürzt und wir mussten ins Krankenhaus, in die Notaufnahme.

2. Letztes Jahr, _ _ wir nach Österreich gefahren sind, ist fast unser Haus abgebrannt. (die Aufgabe Nummer 3).

In dieser Aufgabe gab es 12 Personen, die falsch geantwortet haben. Die Deutschstunden haben mit der Konjunktion ,,wenn” geantwortet. In dieser Satz gibt es ein Adverb der Konjunktion ,,als”. Das Adverb ist „letztes Jahr” mit dem Konjunktion „als” angewendet und mit Präteritum benutzt wird. Das ist eine einmalige Handlung. Obwohl in dieser Aufgabe das Partizip Perfekt benutzt wird muss die Konjunktion ,als” angewendet werden.

Die richtige Antwort ist:

Letztes Jahr, als wir nach Österreich gefahren sind, ist fast unser Haus abgebrannt.

3. Und errinerst du dich noch an die Schwierigkeiten an der Grenze, wir in die Türkei wollten und mein Vater seinen Ausweis vergessen hat? (die Aufgabe Nummer 5).

In dieser Aufgabe gab es 23 Personen, die falsch geantwortet haben. Die Deutschstunden haben mit der Konjunktion ,,wenn” geantwortet und das ist falsch. In dieser Satz gibt es kein Adverb der Konjunktion ,als”. Der Satz beschreibt einmalige Handlung. Obwohl in dieser Aufgabe das Partizip Perfekt benutzt wird, muss die Konjunktion ,als” angewendet werden.

Die richtige Antwort ist:

Und errinerst du dich noch an die Schwierigkeiten an der Grenze, als wir in die Türkei wollten und mein Vater seinen Ausweis 
vergessen hat?

\section{Teil II}

1. Ich habe kein Wort verstanden, ich das erste Mal in Deutschland war. (die Aufgabe alphabet a)

In dieser Aufgabe gab es 6 Personen, die falsch geantwortet haben. Die Deutschstunden haben mit der Konjunktion ,wenn” geantwortet und das ist falsch. In diesem Satz gibt es ein Adverb der Konjunktion „als”. Das Adverb ist „erste Mal” mit dem Konjunktion ,als” angewendet und mit Präteritum benutzt wird. Das ist eine einmalige Handlung. Obwohl in dieser Aufgabe das Partizip Perfekt und Präteritum benutzt wird, muss die Konjunktion ,als” angewendet werden.

Die richtige Antwort ist:

Ich habe kein Wort verstanden, als ich das erste Mal in Deutschland war.

\section{Teil III}

1. sie zwei Jahre alt war, starb ihr Vater (die Aufgabe Nummer 1).

In dieser Aufgabe gab es 9 Personen, die falsch geantwortet haben. Die Deutschstunden haben mit der Konjunktion „wenn” geantwortet und das ist falsch. In diesem Satz gibt es ein Adverb der Konjunktion ,,als”. Das Adverb ist „zwei Jahre alt war" mit dem Konjunktion ,als” angewendet und mit Präteritum benutzt wird. Das ist eine einmalige Handlung. In dieser Aufgabe wird das Präteritum benutzt.

Die richtige Antwort ist:

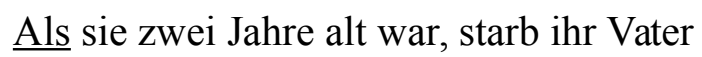

2. Die wollte sie dann aber freier erziehen, sie selbst erzogen worden war; denn an ihre eigene Kindheit dachte sie schon damals nicht so gern zurück. (die Aufgabe Nummer 6).

In dieser Aufgabe gab es 22 Personen, die falsch geantwortet haben. Die Deutschstunden haben mit der Konjunktion ,wenn” geantwortet und das ist falsch. In diesem Satz gibt kein Adverb das zur Konjunktion ,als” gehört. Das ist eine einmalige Handlung. 
Der Satz ist: denn an ihre eigene Kindheit dachte sie schon damals nicht so gern zurück. Der Grund sind, dass man nur einmal Kind und einmal Kindheit waren. In dieser Aufgabe benutzt das Präteritum.

Die richtige Antwort ist:

Die wollte sie dann aber freier erziehen, als sie selbst erzogen worden war; denn an ihre eigene Kindheit dachte sie schon damals nicht so gern zurück.

\section{Die Konjunktion ,Wenn”}

Betreffend der Konjunktion ,wenn” wurden von den Deutschstudenten insgesamt 199 (51,28\%) Fehler gemacht. Viele Fehler haben die Deutschstudenten bei der Anwendung der Konjunktion ,wenn” gemacht. Die Deutschstudenten verstehen die Anwendung der Zeitform der Konjunktion ,wenn” nicht. Die Konjunktion „wenn” wird im Präsens, Präteritum und Partizip Perfekt angewendet und die Konjunktion „wenn” wird für mehrmalige Handlungen benutzt. Die Konjunktion „,wenn” kann im Präteritum angewendet werden, wenn eine wiederholte Handlung in der Vergangenheit beschreiben wird. Die Deutschstudenten können zwischen der Bedeutung der Konjunktionen ,als" und „wenn” nicht unterscheiden. Die Deutschstudenten können zwischen dem Adverb für Konjunktion ,als” oder „,wenn” nicht unterscheiden. Das Adverb der Konjunktion ,,wenn” sind ,immer”, ,,jedes Mal”. Die Deutschstudenten verstehen die Bedeutung der Sätze nicht, die nur einmalige Handlungen oder mehrmalige Handlungen beschreiben. Deshalb machen die Deutschstudenten Fehler im Satz.

Diese Fehler gibt es in den Aufgaben Nummer 2,7 im ersten Teil, bei Buchstabe $b$ im zweiten Teil, Nummer 4,7,8,9,10 im dritten Teil. Die meisten Fehler wurden bei Nummer 7 im dritten Teil (38 Fehler) und die wenigsten Fehler in Nummer 8 im dritten Teil (14 Fehler) gemacht.

\section{Teil I}

1. Aber jedes Mal, ___ wir wieder nach Hause gekommen sind, haben meine Eltern gesagt: „Klasse Urlaub!” Na ja, noch zwei Wochen. (die Aufgabe Nummer 7). 
https://jurnal.unimed.ac.id/2012/index.php/studia/index

In dieser Aufgabe gab es 29 Personen, die falsch geantwortet haben. Die Deutschstunden haben mit der Konjunktion ,als" geantwortet und das ist falsch. In diesem Satz gibt es das Adverb der Konjunktion „wenn”. Das Adverb ist „,jedes Mal” mit dem Konjunktion ,wenn” angewendet und das Partizip Perfekt benutzt wird. Es wird eine mehrmalige Handlung beschreiben. In dieser Aufgabe wird das Partizip Perfekt benutzt.

Die richtige Antwort ist:

Aber jedes Mal, wenn wir wieder nach Hause gekommen sind, haben meine Eltern gesagt: „Klasse Urlaub!” Na ja, noch zwei Wochen.

\section{Teil II}

1. Typisch Papa! Immer wir in Urlaub gefahren sind, hat er etwas vergessen. (die Aufgabe alphabet b).

In dieser Aufgabe gab es 27 Personen, die falsch geantwortet haben. Die Deutschstunden haben mit der Konjunktion ,,als" geantwortet und das ist falsch. In diesem Satz gibt es das Adverb der Konjunktion ,wenn”. Das Adverb ist ,immer” mit der Konjunktion ,wenn” angewendet und das Partizip Perfekt benutzt wird. Es wird eine mehrmalige Handlung beschreiben. In dieser Aufgabe wird das Partizip Perfekt benutzt.

Die richtige Antwort ist:

Typisch Papa! Immer wenn wir in Urlaub gefahren sind, hat er etwas vergessen.

\section{Teil III}

1 . zum Beispiel die Mutter nachmittags schlief, durften die Kinder nicht laut sein und spielen. (die Aufgabe Nummer 4).

In dieser Aufgabe gab es 16 Personen, die falsch geantwortet haben. Die Deutschstunden haben mit der Konjunktion ,als" geantwortet und das ist falsch. In diesem Satz gibt es kein Adverb der Konjunktion „wenn”. Es wird eine mehrmalige Handlung beschreiben. Obwohl in dieser Aufgabe das Präteritum benutzt wird, ist die richtige Konjunktion ,wenn”.

Die richtige Antwort ist:

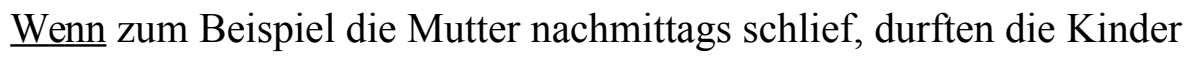
nicht laut sein und spielen. 

zum Beispiel Besuch im Hus war, dann mußten die Kinder gewöhnlich in ihrem Zimmer bleiben und ganz ruhig sein. (die Aufgabe Nummer 7).

In dieser Aufgabe gab es 38 Personen, die falsch geantwortet haben. Die Deutschstunden haben mit der Konjunktion ,als” geantwortet und das ist falsch. In diesem Satz gibt es kein Adverb der Konjunktion ,wenn”. Es wird eine mehrmalige Handlung beschreiben. Obwohl in dieser Aufgabe das Präteritum benutzt wird, ist die richtige Konjunktion ,wenn”.

Die richtige Antwort ist:

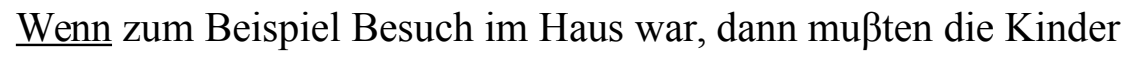
gewöhnlich in ihrem Zimmer bleiben und ganz ruhig sein.

\section{Die Schlussfolgerung}

Basierend auf dem Ergebnis der Untersuchung werden die folgenden Schlussfolgerung gezogen: Es gibt zwei Arten von Fehlern, die von den Deutschstudenten bei der Anwendung der Konjunktionen ,als” und ,wenn” gemacht werden, das sind Fehler, bezüglich 1) der Konjunktion ,,als” (189 Fehler, 48,71\%), 2) der Konjunktion „wenn” (199 Fehler, 51,28\%). Ausgehend von der Fehleranalyse werden am meisten Fehler bezüglich der Konjunktion „wenn” (199 Fehler, 51,28\%) gemacht.

Die Ursache der Fehler von den Deutschstudenten im dritten Semester im Jahrgang 2017/2018 an der Fremdsprachenabteilung der staatlichen Universität Medan bei der Anwendung der Konjunktionen ,als” und „wenn” sind 1) Die Deutschstudenten verstehen die Anwendung der Zeitform der Konjunktionen ,,als” und „wenn” nicht, 2) Die Deutschstudenten können zwischen die Bedeutung der Konjunktionen ,als” und „wenn” nicht unterscheiden, 3) Die Deutschstudenten können zwischen die Adverbien für die Konjunktionen ,als” oder ,wenn” nicht unterscheiden, 4) Die Deutschstudenten verstehen die Bedeutung der Sätze nicht, die nur einmalige Handlungen oder mehrmalige Handlungen beschreiben.

\section{LITERATURVERZEICHNIS}

Bahri, Saiful and Sugeng, Bambang. 2008. Difficulties in Writing in Vocabulary and Grammer of The Second Year Students of SMPN I Selong East Lombok, West 
https://jurnal.unimed.ac.id/2012/index.php/studia/index

Nusa Tenggara in The School Year 2008/2009. Logat:Jurnal UNY, I (2) 1, 8-10, 12-15. (letzter Aufruf: 02.04. 2017).

Bock, Heiko. dkk. 1997. Themen Neu 2. Jakarta: Katalis.

Chaer, Abdul. 2005. Linguistik Umum. Jakarta: Rineka Cipta.

Götze, Lutz. 2004. Grammatik der Deutschen Sprache. Köln: Wissen Media GmbH

Grandi, Nicoletta. 2004. Herzlich willkommen. Berlin: Druckhaus Langenscheidt.

Griesbach, Heinz. 1970. Deutsche Grammatik im Überblick. München: Max Hueber.

Hilpert, Silke. dkk. 2007. Schritte International 5. München: Hueber.

Kleppin, Karin. 2000. Fehler und Fehler Korrerktur. Berlin: Druckhaus Langenscheidt.

Parera, Jos. 1997. Linguistik Edukasional. Jakarta: Erlangga.

Tarigan, Guntur dan Tarigan. 2011. Pengajaran Analisis Kesalahan Berbahasa. Bandung: Angkasa.

Veijonen, Jaana. 2008. Zur Fehleranalyse. Eine empirische Untersuchung von Wortstellungs und Verbrektionsfehlern in deutschen Aufsätzen finnischer Gymnasiasten.Abrufbarunter:

https://tampub.uta.fi/bitstream/handle/10024/79240/gradu02849.pdf?sequence=1. (letzter Aufruf: 02.04. 2017).

https://books.google.co.id/books?id=wSCLBgAAQBAJ\&pg=PA15\&dg=brief

+ hallo + als + wenn + ich\&source $=$ bl\&ots $=3 Z$ UHQFFslM\&sig $=$ dpxk9FJNSzD3189

R2KdRWzzflY\&hl=id\&sa=X\&ved=0ahUKEwiu6azS1qbUAhVJL18KHV4qBel

Q6AEINTAI\#v=onepage\&q\&f=false. (letzter Aufruf: 10.04.2017).

https://is.muni.cz/el/1421/jaro2012/NJI_072/Die_Konjunktion.pdf. (letzter Aufruf: 10.04.2017).

http://mein-deutschbuch.de/grammatik.html. (letzter Aufruf: 04.04.2017).

https://is.muni.cz/el/1421/jaro2012/NJI_072/Die_Konjunktion.pdf. (letzter Aufruf: 10.04.2017).

Biografie des Authors:

Meilyn Sarah Pangaribuan ist Alumni des Deutschprogramms von Unimed, Medan 
